\title{
Intervenciones reparatorias estatales para el tratamiento de prácticas abusivas sexuales basadas en una mirada integrativa
}

\author{
State Reparative Interventions for the Treatment of Sexual \\ Abuse Practices Based on an Integrative Perspective \\ Intervenções de reparação estatal para o tratamento de \\ práticas abusivas sexuais baseadas em uma abordagem \\ integrativa
}

Carolina Alejandra Muñoz Retamal*

\section{RESUMEN}

Este artículo realiza una propuesta metodológica basada en la Palabras clave: experiencia clínica, que pretende ser un aporte al diseño de inprácticas abusivas tervenciones enfocadas a brindar tratamiento a niños, niñas y adolescentes que han ejercido prácticas abusivas de carácter sexual (PAS), en el marco de la oferta programática estatal de Chile, la cual se basa, en su mayoría, en modelos ecológicos y multifactoriales para explicar y tratar el fenómeno. En este contexto, se plantean ciertas brechas en relación con el diseño e implementación de intervenciones más personalizadas. Se propone, entonces, un recorrido por los principales postulados psicoanalítisexuales; género; psicoterapia infantojuvenil; cultura; legalidad; abuso sexual; psicoanálisis; enfoque integrativo, PAS. cos, que sirvan para comprender la incidencia y la significación de la ley, tanto en el individuo como en la cultura, con el objetivo de diseñar intervenciones que contemplen estas variables y que sean consecuentes, a su vez, con el modelo ecológico.

\begin{abstract}
This article offers a methodological proposal based on clinical experience, which aims to contribute to the design of interventions focused on providing treatment to children and adolescents who have practiced abusive practices of a sexual nature (PAS), within
\end{abstract} Key words: Sexual abuse practices; Gender; 
the framework of the Chilean government's programmatic offer, which is based mostly on ecological and multifactorial models to explain and treat the phenomenon. In this context, certain gaps arise in relation to the design and implementation of more personalized interventions. It is proposed, then, a review of the main psychoanalytic postulates that serve to understand the incidence and significance of the law both in the individual and in the culture, with the aim of designing interventions that contemplate these variables and that are also consistent with the ecological model.

\section{RESUMO}

Este artigo apresenta uma proposta metodológica baseada na experiência clínica, que visa contribuir para o desenho de intervenções voltadas ao tratamento de crianças e adolescentes que tenham exercido práticas abusivas de natureza sexual (PAS), no âmbito da oferta programática estatal do Chile, a qual está baseada, em sua maioria, em modelos ecológicos e multifatoriais para explicar e tratar o fenômeno. Neste contexto, são expostas certas lacunas em relação à concepção e implementação de intervenções mais personalizadas. Propõe-se, então, uma revisão dos principais postulados psicanalíticos que sirvam para compreender a incidência e o significado da lei de uma perspectiva tanto individual quanto cultural, com o objetivo de elaborar intervenções que contemplem estas variáveis e que sejam coerentes, ao mesmo tempo, com o modelo ecológico.
Child and Youth Psychotherapy; Culture; Legality; Sexual abuse; psychoanalysis; integrative approach, PAS.

Palavras-chave: práticas abusivas sexuais; gênero; psicoterapia infantojuvenil; cultura; legalidade; abuso sexual; psicanálise; abordagem integrativa, PAS. 


\section{Introducción}

El presente escrito surge desde el interés por la incidencia de la significación de la ley dentro de la psique de los individuos en sociedad, teniendo como perspectiva teórica la tríada «individuo-ley-cultura» en forma triangular, graficando con esto la interdependencia de sus elementos y la casi imposibilidad de pensar uno sin el otro.

Con ello se reflexiona en torno a esta incidencia desde la visión particular de niños, niñas y adolescentes que trasgreden leyes universales de regulación social e interacción sexual permitidas entre los individuos en la cultura actual. Esto basado en la experiencia profesional como psicóloga clínica en el programa reparatorio de la oferta programática de Protección Especializada del Servicio Nacional de Menores, Programa PAS, el cual brinda tratamiento a niños, niñas y adolescentes (entiéndase NNA) que presentan prácticas abusivas sexuales (entiéndase PAS).

De acuerdo con esto, se plantea entonces una propuesta metodológica basada en postulados psicoanalíticos respecto de la operancia interna de la ley, la cual, además, pueda integrarse dentro de los modelos conceptuales de los programas PAS que, en la actualidad, están basados en un enfoque ecológico y multifactorial principalmente.

Junto con ello, y de manera transversal, la presente reflexión teórico-clínica pretende promover a expandir las fronteras conceptuales del terapeuta, para que la teoría, lejos de limitar, siente las bases de un conocimiento práctico que ayude a desplegar herramientas clínicas creativas y basadas en el encuentro mismo entre terapeuta y niño/ entorno, permitiendo abordar el hecho lesivo desde los ojos de su ejecutor y, desde una postura clínica, intervenir más eficazmente en su mundo.

\section{Prácticas abusivas sexuales en NNA y su abordaje en el contexto chileno actual}

Existen diversas prácticas sexuales consideradas abusivas que varían enormemente según su calidad, frecuencia y aparición; no obstante, existe consenso profesional respecto de su condición abusiva en los casos en que existe una relación sexual asimétrica entre dos o más personas, ya sea en relación con la edad, capacidades cognitivas y/o 
físicas, uso de coerción, etc., y no solo con relación al uso de violencia física.

El abuso sexual es un objeto de estudio especialmente atingente a las ramas de la psicología que trabajan con temáticas de infancia y adolescencia, ya que la ontogenia de este fenómeno, en términos individuales, se sitúa en estas etapas del desarrollo. De hecho, actualmente, se estima que los jóvenes son responsables de un tercio de los abusos sexuales hacia otros NNA (ONG Paicaví y Aldeas infantiles, 2014, p. 4) Por otra parte, los estudios de José Diaz Morfa (2003) muestran que alrededor del $20 \%$ de las violaciones y $50 \%$ de los abusos sexuales han sido realizados por adolescentes, y aproximadamente el 50\% de los adultos con conductas de ofensa sexual ha cometido las primeras agresiones durante su juventud. Respecto de las cifras nacionales, no existe un registro unificado, sumado además a una importante cifra de casos no denunciados. Sin embargo, según las estadísticas que entrega el Ministerio Público, en 2013 se registraron 1.092 denuncias por delitos sexuales cometidos por adolescentes en el marco de la Ley de Responsabilidad Penal Adolescente (LRPA), siendo probable que esta cifra corresponda solo a un $10 \%$ del total de los casos (Deprode, Sename, 2019).

La agresión sexual, en tanto delito, se encuentra tipificada en la ley penal según su calidad y, por tanto, también lo es bajo la LRPA 20.084, siendo el Servicio Nacional de Menores, SENAME, la entidad encargada de administrar las sanciones decretadas por el Tribunal de Garantía. En Chile existe un amplio trabajo investigativo respecto del estudio y diseño de intervenciones enfocadas a la población de adolescentes que cometen todo tipo de delitos. Un claro ejemplo es el Modelo Multidimensional de Intervención Diferenciada con Adolescentes (MMIDA), que ha contado con el respaldo de diversas instituciones públicas y privadas, y que responde a una investigación sistemática y exhaustiva de las características psicológicas, factores de riesgo y comportamiento delictivo de los infractores de ley juveniles (Pérez-Luco et al., 2014).

A través del modelo de trayectorias delictivas que arroja el MMIDA, se logra realizar una diferenciación de grupos según la especificidad de los comportamientos exhibidos, definiéndose la trayectoria de delincuencia transitoria versus una delincuencia persistente, siendo éstas las más estudiadas y con un diseño interventivo consistente, 
pues es donde se agrupa la mayor población de infractores juveniles. Sin embargo, se diferencia un tercer tipo, el de la delincuencia compleja y, dentro de éste, un subgrupo minoritario de adolescentes que ha realizado ofensas sexuales, entendido como un comportamiento aislado e incomprensible en un contexto habitual de conducta socialmente adaptativa. Este grupo presenta características heterogéneas que crean y mantienen el funcionamiento delictivo, y que acrecientan el riesgo y la gravedad del delito, entre los que se encuentran: trastornos de salud mental, traumas infantiles crónicos, relaciones vinculares ambivalentes, alteraciones en patrones de personalidad y presencia de conflictos emocionales y conductuales (Pérez-Luco et al., 2015).

Las características mencionadas llaman aún más la atención cuando se tienen en cuenta los factores etiológicos que la literatura establece respecto del comportamiento abusivo en estas etapas del desarrollo, entre los que se encuentran: victimización infantil, modelaje de la sexualidad a través del abuso, violencia y coerción, y alteración del rol de protección, cuidado y guía del grupo familiar (Friedrich, 2003), siendo más clara la relevancia de abordar de manera diferenciada la estrategia de rehabilitación de adolescentes que cometen delitos sexuales.

De hecho, a la luz de estos datos, en Chile se generó una importante diferenciación en relación con el análisis y tratamiento para este grupo minoritario claramente diferenciado, por lo que Sename implementa intervenciones específicas dentro de los programas de la línea de LRPA ya existentes. Sin embargo, en 2002 Sename implementa un proyecto psicosocial especial para brindar tratamiento a NNA que presentan prácticas abusivas sexuales - Programa PAS -, con la notable diferencia de que éste no se inserta dentro de la oferta programática de la línea de LRPA sino en la de Protección Especializada que ejecuta la misma institución pero que depende en Tribunal de Familia, siendo estos programas destinados a "(...) otorgar intervención reparatoria a NNA víctimas de graves vulneraciones de derechos u otras problemáticas que atentan gravemente contra su normal desarrollo" (Deprode, Sename, 2019, p. 3). La población varía entre los 10 y 17 años, es decir, tanto NNA inimputables como imputables, en tanto estos últimos respondan a variables claramente más ligadas a la vulneración de derechos.

Por lo tanto, cuando se efectúa un tratamiento psicoterapéutico de esta índole, es fundamental tener en cuenta que la conducta abu- 
siva es, muy probablemente, una sintomatización de la historia de las vulneraciones de NNA, las cuales, lejos de ser solo historia, se manifiestan dinámicamente en la emisión de esta conducta actualizada que incluye una nueva vulneración hacia otro, para pasar entonces, el niño/o ejecutor de PAS, a ser un victimario en relación a los roles de esta interacción siempre asimétrica, y, por tanto, a conformarse como un trasgresor de una ley fundamental que ordena a la cultura, en un círculo vicioso y de límites ya difusos entre un/a vulnerado/a y un/a vulnerador/a. De allí, la importancia de contar con un tratamiento estatal oportuno y efectivo respecto de esta problemática socioculturalmente tan dinámica.

La implementación del programa PAS ha permitido, no solo aumentar la visibilidad de esta población minoritaria, sino, además, visibilizar una inminente necesidad de continuar avanzando en estudios investigativos que permitan conocer a este sector en mayor profundidad. En efecto, esto se ha logrado en mayor grado gracias a la sistematización de las características psicométricas de los instrumentos DASH-13 y ERASOR 2.0, que miden variables para determinar factores protectores y de riesgo en la población adolescente chilena que ejerce PAS, respectivamente.

En relación con el abordaje psicoterapéutico de este fenómeno, los organismos privados que opten por postular a la licitación de un proyecto PAS (a través del sistema de OCAS u Organismos Colaboradores de Sename) deben someterse a uno o más modelos explicativos acerca del surgimiento de las prácticas abusivas sexuales que se dan a conocer en las bases técnicas del proyecto, entre las que se encuentran: teorías multifactoriales (modelo multisistémico, modelo comprensivo, modelo de las cuatro precondiciones de Filkenhor y modelo de vida satisfactoria); teorías monofactoriales (modelo biológico, modelo psicoanalítico, modelo feminista, modelo conductual y modelo sistémico), y modelos descriptivos o de proceso (ciclo del abuso destinado a evitar recaídas). No obstante, en el mismo documento se señala que se observó que, en la mayoría de los programas PAS, prevalece un modelo ecológico o ecosistémico, con énfasis en la existencia de una matriz cultural en que se inscriben las prácticas abusivas (Deprode, Sename, 2019).

Es importante especificar que este modelo generalizado percibe al ambiente social como un conjunto de estructuras seriadas en varios 
niveles, en los que cada uno contiene al otro. Bronfenbrenner (1987), autor de la teoría, denomina a esos niveles el microsistema (usualmente la familia), el mesosistema (interrelaciones entre dos o más entornos directos del sujeto), el exosistema (contextos más amplios que incluyen activamente al sujeto) y el macrosistema (cultura y subcultura). Sin embargo, posteriormente, en 1994, la teoría se reformula, planteando el desarrollo como un fenómeno continuo de cambio de las características biopsicológicas del ser humano, tanto de los grupos como de los individuos. El elemento crítico de este modelo actualizado es el de abordar la experiencia no solo desde sus propiedades objetivas, sino también las que son subjetivamente experimentadas por las personas que viven en ese ambiente (Frías-Armenta et al., 2003).

Ahora bien, respecto del diseño de intervenciones en PAS en el contexto nacional, han habido un trabajo corporativo y mancomunado en esta línea, siendo posible encontrar el manual CAS-R (Programa de tratamiento para el control de la agresión sexual) y el diseño de intervenciones psicosociales en PAS de la ONG Paicaví (específicamente del Centro Trafún de la misma institución). No obstante, se considera que aún sigue habiendo una escasez de tecnología psicosocial para un abordaje eficiente (Pérez-Luco et al., 2015), y que las intervenciones actuales presentan dificultades para abordar casos más graves, y deficiencias en su rigurosidad (Valenzuela y Escudero, 2013).

Por otra parte, si bien estos diseños de intervención ponen énfasis en una mirada global, muy necesaria para comprender el problema en su totalidad y desde una visión crítica y comprensiva, se observa algunas limitaciones en su implementación técnica en la clínica, ya que, muchas veces, éstas se limitan al uso de manuales o materiales prediseñados que presentan el riesgo de brindar un tratamiento que cae en lo mecánico y, con esto, que se reste esfuerzo y dedicación a la tarea de reflexionar y pensar en torno al caso particular y invisibilice a NNA, perdiéndose las ganancias de crear estrategias basadas en los recursos de la misma experiencia que entrega el abordaje del caso.

Para que esto no ocurra, se propone incluir, estratégicamente, intervenciones basadas en perspectivas más individuales que pudieran complementar a las intervenciones actuales, para hacer una especie de efecto lupa que ayude a comprender mejor a la totalidad. Con base en esto, se propone entonces presentar los postulados psi- 
coanalíticos como una herramienta teórica que inspire a diseñar intervenciones clínicas basadas en el análisis individual del niño/a y su entorno, ya que esta perspectiva, si bien individual, comprende la incidencia crucial de la cultura y (la familia) dentro de la psique, por lo que es posible encontrar ideas que se homologan y que pudieran complementarse.

Por otro lado, el enfoque psicoanalítico también se vuelve especialmente relevante para el propósito, ya que explica la constitución del psiquismo humano sobre la base del desarrollo psicosexual basado en conceptos claves que se vinculan directamente con la comisión de prácticas abusivas sexuales — como los de ley, cultura y sexualidad -, y al proceso de interiorización de normas fundamentales de regulación social, principalmente sexuales y de agresión. Sexualidad, agresión, amor, placer y control se considerarán como instancias claves que pondrán en juego los límites entre la individualidad y la cultura, a través de la implementación de una ley común.

Ante ello, cabe preguntarse: ¿cómo puede una mirada psicoanalítica contribuir al diseño de intervenciones bajo el marco actual de los programas PAS - Prácticas Abusivas Sexuales-, basados en un modelo ecológico?

Para responder a lo anterior, y según postulados psicoanalíticos, se dará a conocer el recorrido psicológico que realiza el individuo en las primeras etapas del desarrollo en relación con la tramitación de sus pulsiones, la incorporación de la leyes relativas a la prohibición del incesto y del asesinato (resaltando la concordancia entre la teoría y los resultados de investigaciones respecto de la función social del neocórtex), y la introducción a una cultura predominantemente patriarcal (incorporando una perspectiva de género), lo cual explica, en no menor medida, la prevalencia de PAS como mayormente masculinas.

Basada en este recorrido, se desarrollará una propuesta de intervención que contemple todas las variables mencionadas, promoviendo que el terapeuta pueda comprender y analizar la incidencia de estos factores individuales en relación con la tramitación de la PAS y que, además, se pueda incluirlos fácilmente en intervenciones basadas en un modelo ecológico o multifactorial. 


\section{Proceso de introyección de la ley y entrada a la cultura desde el psicoanálisis}

Cada vez es mayormente aceptada la idea de que niños y niñas vivencian su sexualidad desde el nacimiento, existiendo poca información epidemiológica al respecto. Aun así, se tiene el ejemplo de la masturbación - todo acto auto estimulatorio que tiende a producir satisfacción sexual—, que pertenece a un espectro amplio de comportamiento sexual normal a cualquier edad, describiéndose incluso casos de masturbación intrauterina (Peinado, 2010, p. 1).

Según postulados psicoanalíticos freudianos, la condición sexual y la regulación de su energía o líbido tienen una incidencia crucial en el proceso de socialización de los individuos, sobre todo en las primeras etapas del desarrollo, ya que en ellas se definirá, en gran medida, la vida sexual y socioafectiva que se tendrá en etapas posteriores. Este proceso de autoregulación opera mediante la interiorización (introyección) de un complejo sistema impuesto por la cultura, enfocado en la adquisición de normas y prohibiciones básicas que pondrán en juego la tramitación de la energía sexual, el instinto de supervivencia y, con ello, la afectividad (Freud, 1978).

En relación con la actividad intrapsíquica infantil en las primeras etapas del desarrollo, la psicoanalista Melanie Klein (1932) describe cómo el neonato tramita sus sentimientos de dependencia física, sexual y afectiva desde diferentes fases, según su capacidad de diferenciación con la madre. Instintivamente, vivenciará el amor más profundo hacia su madre-pecho (objeto entonces parcial) pero también vinculará a ella las sensaciones displacenteras, generando sentimientos ambivalentes de amor, odio y destrucción; por lo que llama a ésta, la etapa paranoide. En esta etapa no existe una diferenciación entre sí mismo y la madre-entorno, ni menos entre el instinto de supervivencia, las necesidades sexuales (placer principalmente por succión) y las afectivas. Posteriormente, y si —como ocurre comúnmente- el bebé se descubre diferente a su madre, abandona la etapa paranoide, surgiendo sentimientos depresivos vinculados a una conciencia temprana de la posibilidad de pérdida del objeto de amor y ansiedad de separación (Klein, 1932).

Pues bien, el deseo sexual materno del niño/a, cargado de afectividad y sentimientos agresivos de dañar a quien se interponga en el logro 
de sus satisfacciones, debe reprimirse, accediendo, a cambio, al pase cultural y a beneficios sociales. Es así que, como condición cultural, el niño introyecta dos leyes sociales básicas: la prohibición del incesto y la prohibición de asesinato, a través de la instalación de una instancia psíquica autoregulada por el sentimiento de culpa (súper yo como autoridad introyectada), desde la que surgen todos los imperativos categóricos de lo prohibido (Freud, 1930), lográndose además que el niño/a reprima sus impulsos sexuales hasta la pubertad y solo regulado por la ley de la exogamia y la consensualidad (Freud, 1978).

La cultura se origina entonces como una forma de regular las relaciones entre los individuos para lograr vivir en comunidad. Siguiendo los postulados anteriores, el costo de vivir en una cultura implica la renuncia al propio campo de satisfacciones - la imposibilidad de la consecución del principio del placer- lo que genera un individuo en constante malestar en la cultura (Freud, 1930). De aquí que muchas patologías que Freud describirá a lo largo de su obra estén basadas en cómo el individuo vivencia y expresa inconscientemente su malestar, pues donde hay una prohibición, está la represión de un deseo que genera displacer.

\section{Vinculación entre los postulados psicoanalíticos y aportes de las neurociencias}

Llama la atención cómo el psicoanálisis, surgido en 1920, describe el hito del desarrollo de la adquisición del lenguaje verbal (digital) como la vía de comunicación más sofisticada por la que se introyectará paulatinamente el sistema de normas y prohibiciones (en el que el padre toma protagonismo), en desmedro de un lenguaje únicamente análogo. Esto porque lo anterior se condice con resultados de investigaciones contemporáneas; en efecto, el lenguaje tiene directa relación con el desarrollo de la neocorteza cerebral o neocortex, una compleja estructura dinámica a la cual se vinculan variadas habilidades cognitivas y sociales concernientes no solo al lenguaje y decodificación de símbolos, sino a todo lo que el individuo considera como comportamiento adecuado. De hecho, es posible inferir que el desarrollo del neocortex es la estructura fisiológica que permite que el individuo se organice culturalmente sobre la base de leyes y adquiera un desarrollo moral. En efecto, un estudio realizado por Aiello y Dunbar (1998) arroja una relación directa entre el 
tamaño del neocortex y la cantidad de miembros que forman los grupos sociales, lo cual conlleva, a su vez, la posibilidad de relacionarse sobre la base de dinámicas cada vez más complejas, como son la cooperación y la competencia, entre otras (Arsuaga y Martínez, 1998).

\section{Consideraciones de la teoría psicoanalítica desde una perspectiva de género}

La teoría del psicoanálisis merece ser contextualizada, pues se considera que el autor efectivamente plantea el desarrollo de un psiquismo patriarcal, como efecto de una cultura que se origina del mismo modo (graficada filogenéticamente a través del mito de la horda primitiva), siendo esto un problema estructural y, con ello, psicológico. En efecto, dentro del campo de las ciencias sociales, se establece como uno de los principios fundamentales del origen de la civilización la obligatoriedad de la exogamia y la conjunta prohibición del incesto. Existen diferentes teorías acerca del origen de la prohibición, principalmente económicas y de género, siendo la mujer, históricamente, utilizada como un objeto de intercambio y discriminada del mundo público.

De hecho, históricamente, el representante cultural encargado del proceso de introyección de la ley e ingreso a la cultura ha sido el padre (o sustitutos). A pesar de que el vínculo que la madre establezca con el niño sentará las bases de su personalidad, ella es paulatinamente desplazada por la figura paterna. Finalmente, el padre se transforma generalmente en la principal figura de autoridad para el niño/a y, además, posiblemente, una figura de admiración en tanto representante cultural. Lo anterior sugiere cómo al niño/a se le presenta el espacio cultural como un anhelo de mayor estatus y que tiene al género masculino como los representantes culturales y legales de mayor jerarquía. Mientras, tradicionalmente, las niñas están llamadas mayormente a perpetuar el orden de lo establecido, y quedan identitariamente vinculadas a significantes subvalorados relativos a lo afectivo (crianza y apego) y al mundo privado (tareas domésticas; del cuerpo). La envidia del pene, caricaturizada hoy en día, grafica posiblemente la conciencia social respecto de los beneficios que otorga la cultura a la masculinidad, y ciertamente no la envidia de una condición biológica.

Consecuencia de esto pudiera ser el hecho de que los hombres cometan sustancialmente más crímenes que las mujeres, extendiéndose 
aún más la brecha en relación con los delitos sexuales, pues no menor es el hecho de que, durante 2019 , se haya registrado un $93 \%$ de población masculina en los programas PAS, en desmedro de un $7 \%$ de femenina. Con esto, el delito sexual se vuelve indiscutidamente un tema de género. Además, según esta ideología patriarcal, es dable que los hombres dependan mayormente de referentes masculinos para su autoregulación e introyección de la autoridad. Esto se vuelve paradojal, pues parece peligroso delegar tareas de autoridad y regulación a quien objetivamente está más ausente. Por ejemplo, según las Estadísticas Vitales 2016 del Instituto Nacional de Estadísticas (INE), en ese año, en Chile, se registraron 231.749 nacimientos, de los cuales 20.138 fueron casos con padres no comparecientes (es decir, de los cuales no existe registro alguno), equivalentes a $8,7 \%$ del total, volviéndose más difusas y claramente más contundentes en número las cifras respecto de padres comparecientes pero ausentes en la crianza, o insuficientemente vinculados.

Como análisis personal, pareciera ser que se menosprecia, en tanto cultura patriarcal, la importancia de la afectividad en cuanto necesidad orgánica (aspecto ligado al mundo femenino), pues, en realidad, todo niño/a desea sentirse integrado y aceptado por su entorno - principal beneficio cultural一, ya que, además de deseos incestuosos y sádicos que se abordarán en la terapia, también subyace un deseo igualmente intrínseco de sentirse amado no solo por una gran y única persona (como lo fuera la madre en etapas tempranas), sino por muchas, como lo es en la adultez en contexto social. Es sobre este sentimiento de pertenencia sobre el cual se propone anclar la intervención en PAS. Además, con todo lo anterior, pareciera ser que el menoscabo de las necesidades afectivas y la delegación de los roles autoritarios al mundo masculino pudieran ser variables que gatillen la perpetuación del abuso y que, por tanto, debieran abordarse igualmente.

\section{Propuestas metodológicas con base en objetivos de intervención en PAS}

Es innegable que el tratamiento tiene un mejor pronóstico cuando se requiere de una intervención mayormente familiar y socio comunitaria, y se interviene, por tanto, sobre los factores ambientales que se encuentran actualmente en juego con los factores psicodinámicos del 
niño/a, y que lo predisponen, a un nivel conductual-situacional, a la ejecución de una PAS. Relevante es que, no pocas veces, se debe trabajar con la historia de vida familiar y/o personal del adulto significativo, ya que en esta historia se encuentran las raíces de un funcionamiento sistémico actual persistente.

Sin embargo, también es innegable que estos factores ambientales y patrones familiares generan patrones en la dinámica intrínseca del niño, transformándose poco a poco en factores individuales que lo predisponen con cada vez mayor independencia de su ambiente para, finalmente, incluso cristalizarse como rasgos de personalidad complejos de modificar a medida que transita por la adolescencia y hacia la adultez. Con esto, el trabajo psicológico con NNA es fundamental, pues la acción abusiva ya ha sido llevada a cabo individualmente.

Con base en el recorrido anterior, se propone realizar una reflexión más personalizada, en tanto la PAS se vuelve mucho más comprensiva cuando se integran variables psicológicas con relación a cómo opera la legalidad, el control y el sentido de lo moral, o la culpa respecto del hecho. Para ejemplificar, el proceso psicológico legal de un niño sano que ejerce una PAS aislada, producto de una vivencia previa (también aislada) de estimulación sexual prematura que alteró su control y regulación de impulsos, puede derivar en que éste genere altos montos de culpabilidad que lo lleven a silenciar o negar la PAS. Esto será muy diferente de la comprensión y abordaje de la PAS de un adolescente que, producto de vulneraciones sexuales intrafamiliares sistemáticas durante su infancia, se desvincule emocionalmente de las víctimas, negando también los hechos. Aunque la ley no opera de la misma forma, la conducta es la misma (niega) y sus mecanismos de defensa, aunque tan diferentes, tienen un mismo fin: obtener menos dolor emocional. Aquello les produce alivio y los aleja de su núcleo traumático. La dolorosa tarea del terapeuta es acercarlos a este núcleo, promover la angustia a través de un cuidadoso acompañamiento y develar con ello abusos sexuales ocurridos incluso hace décadas.

En ese sentido, es importante recordar que todas las intervenciones en PAS tienen como objetivos generales: abordar la interrupción, promover el reconocimiento y responsabilización, resignificar experiencias de vulneración previas y promover estrategias de prevención de riesgo de reocurrencia (Deprode, Sename, 2019). Es importante orde- 
nar el abordaje de estas metas según la especificidad del caso, ya que muchos casos ingresan reconociendo la PAS, pero también muchos de ellos negarán el reconocimiento a toda costa, siendo capaces de reconocerlo solo cuando se les reconozca a sí mismos como víctimas de abusos sexuales anteriores y se empatice con ellos; esto se vuelve eficaz sobre todo cuando se visualizan altos montos de culpabilidad. Sucede que, al develar y resignificar estas experiencias anteriores, éstas se pueden utilizar como variables explicativas para que el caso entienda su propia conducta trasgresora, facilitándose el desarrollo de empatía con la víctima y, con ello, un reconocimiento del hecho abusivo casi por añadidura. De todas formas, si el niño no reconoce, es preciso continuar con el trabajo y e ir evaluando hasta que se sienta preparado.

\section{Analogías para comprender la sexualidad: estrategia psicoeducativa}

Se propone que, como primera estrategia de psicoeducación, el terapeuta muestre al niño/a la naturalidad del deseo sexual humano, habiendo muchas formas para ello, mediante analogías que se pueden evocar a través de imaginerías. De todas formas, antes de esto, se propone reflexionar con los niños acerca del amplio concepto del placer, realizando una lluvia de ideas de las situaciones placenteras diversas.

Metáfora de la planta hasta llegar a los frutos: Se puede realizar a través de una imaginería; se concibe a la sexualidad interna como una planta que crece desde el pubis o raíz hacia arriba, y que debe regarse para que crezca (sexualidad esperada), pero tampoco más de la cuenta para que no se ahogue (conductas problemáticas o abusivas). Con esto, los NNA sienten que tienen derecho a vivenciar su sexualidad, pero regulada por las tendencias de su ciclo evolutivo y leyes simples que se verán a continuación.

Metáfora de la llama de fuego: Otra estrategia de gran ayuda es realizar un papelógrafo con una gran llama (de tendencia circular hasta legar a la punta) que representa a la sexualidad. En la base se está en los 0 años y el grosor es de término medio, pero su calor es muy bajo; luego va creciendo y se vuelve más gruesa en la adolescencia (pubertad) y más caliente, para luego, mientras va subiendo hacia la adultez, ir intensificando su calor, pero también regulando su llama (control y madurez). En cada ciclo, al lado de la llama se escriben edades tenta- 
tivas y las conductas esperadas, desde: que te guste alguien, escribir una carta, darse la mano, pedir pololeo, dar un beso, tocarse, sueños sexualizados, relaciones sexuales, etc. (dependiendo de la edad). Con esto se crea un lenguaje entretenido, en el que enumeran conductas que hacen que la llama crezca más de la cuenta y que el niño/a pueda quemarse; por ejemplo, por excesiva pornografía (conducta problemática) o por el contacto con alguien que tiene una llama más grande y que puede quemarlo (conducta abusiva), por dar ejemplos.

Las tres leyes universales de la sexualidad: herramienta para la interrupción y problematización de PAS

Una vez se ha establecido un vínculo de confianza y complicidad clave, es posible observar, la mayoría de las veces, un niño/a asustado que desea ser aceptado e integrado por la cultura; no obstante, se encuentra conflictuado, pues tiene sobre sí la etiqueta de agresor sexual, la cual es contracultural. Pero el terapeuta, que tiene una amplia visión y conocimiento de la diversidad sexual, y que en todo momento se muestra empático en relación con los impulsos sexuales del niño/a, lo acepta amorosamente, a pesar de lo que haya hecho. Esto lo sorprende. Sintiéndose comprendido, el NNA descansa en el terapeuta, quien viene a tomar un rol de mediador o intermediario entre la cultura y sus propias pulsiones; fuerzas que para él se encuentran en pugna.

Se propone entonces, como forma de interrupción de urgencia, hacer un contrato terapéutico, en el cual el NNA deba acatar el cumplimento de las tres leyes de la sexualidad, basadas en las formas de interacción sexual aceptadas por la cultura, profundizándose en ellas según sus intereses, ideas previas y, sobre todo, el tipo de PAS:

1. Toda interacción sexual debe ser consensuada: La obligatoriedad implica agresión o aplicación de fuerza asimétrica en cualquiera de sus variantes (física, intelectual, de poder, de género, etc.). El consenso mutuo es una forma de relación básica entre los individuos, en todas las formas de interacción. La posibilidad de elección de con quienes nos relacionamos sexualmente es un derecho básico inquebrantable.

2. No debes interactuar sexualmente con un niño/a menor o mayor que tú (de diferente ciclo vital): La asimilación de esta de ley implica una psicoeducación sexual basada en el ciclo vital y en problematizar 
creativamente las consecuencias de una estimulación sexual temprana (a través de la llama). De esta manera, el niño/a comprende por qué no es sano que dos personas en diferentes etapas no se relacionen sexualmente, sino que dentro de otras formas de interacción amorosa.

3. No debes interactuar sexualmente con integrantes de tu misma familia: Es común que las PAS se den en un contexto intrafamiliar, trasgrediendo esta ley primigenia básica que sanciona la cultura, pero que no está penalizada por la ley si es que no hay una trasgresión de alguna de las leyes anteriores, las cuales tienen mayor peso y objetividad a la hora de presentarlas a los NNA. No obstante, es importante socializar esta ley, sobre todo en casos en que existe deprivación sociocultural, tendencia a la normalización de juegos sexualizados, por ejemplo, o hacinamiento como factor de riesgo.

\section{La maqueta como representación simbólica de la cultura:} técnica para la resignificación, problematización y desarrollo de estrategias de prevención de riesgo

Recordando el rol de mediador entre las pulsiones del niño/a y la cultura, el terapeuta propone la construcción de una maqueta, concepto libremente elegido para dar a entender cualquier opción terapéutica con la que el niño/a pueda representar su idea de mundo como metáfora de la sociedad que lo contiene y, junto con ello, se vuelva consiente del entramado social y su funcionamiento. Esta maqueta o montaje puede ser una bandeja de arena, un lienzo, un escenario, técnicas de artes plásticas, etc., pero siempre, una obra. Mediante su construcción, se instruirá al niño/a para que, según su edad, vaya adquiriendo ideas que le permitan representar conceptos como los de cultura (maqueta), sociedad, comunidad, yo, personas, leyes, normas, identidad, empatía, deseos, impulso, control, derechos, deberes, género, competencia, alian$z a$, colaboración, conflicto, etc., mientras se le va dando una idea general del concepto, para que él busque dónde, cuándo y cómo lo representaría. Es importante inducir a una perspectiva de género dentro de la maqueta, debido a la incidencia predominante de esta desigualdad como factor incidente.

Diversas analogías son útiles para ayudar al niño/a incorporar estos conceptos, que le brindarán una idea global de mundo en un 
lenguaje propio. Para dar algunos ejemplos, en el caso de preadolescentes ha sido de gran ayuda el psicoeducarlos e intervenir con ayuda de la analogía de las leyes del tránsito, con autos que simbolizan personas, mostrando que el objetivo principal de las leyes es que éstos no choquen entre sí y puedan transitar libremente por el espacio cultural dado (delimitado dentro del juego, según la técnica). Esta es una idea básica para comprender el objetivo de la ley desde su función de utilidad. Como idea, se puede señalar al niño/a: "a veces voy muy atrasado al trabajo (voy maniobrando mi auto) y quisiera pasar la luz roja y así llegar más rápido. ¿Qué pasa? (choque). ¿Y qué pasaría si voy relajado pero el auto que viene atrás está muy atrasado? Otro ejemplo: ¿cómo utilizo la bocina? (Utilizar el recurso de la simbología). ¿Qué puedo hacer si me quedo sin bencina? ¿Cómo reacciono frente a un choque y cómo puedo ayudar? ¿Para qué sirve el límite de velocidad? ¿Qué pasa si me excedo? Yo soy este auto, pero ¿qué pasa si de pronto soy éste? Buses, bencina, dinero, peatones, parques y plazas, escuelas, poblaciones, casas, habitaciones, hasta elementos naturales son una oportunidad para jugar con el niño/a mientras se lo/a educa y además se conoce lo que necesita.

En este sentido, mientras se interviene para que el niño/a se integre en la cultura de modo más adaptativo y comprenda más gráficamente qué consecuencias tienen las acciones que él mismo ejerce, también se va indagando acerca de la tramitación de sus pulsiones sexuales y en cómo falla la operación de la ley introyectada. Esto ya que, en este espacio, tendrá la libertad de desplegar patrones respecto de cómo se desenvuelve en sociedad y sus ideas acerca de las interacciones sociales; además de dar cuenta, simbólicamente, de la forma en cómo ha vivenciado situaciones de vulneración. En la práctica, nos encontraremos en terapia con diversos choques de autos o caos culturales expuestos por el niño/a en sesión, los cuales se van resignificando a medida que el terapeuta influye sutil, espontánea y acertadamente en el escenario terapéutico, comenzando el niño/a a estructurar la maqueta o el montaje utilizado, e introyectando la idea de que él mismo está inmerso en la maqueta (cultura).

La misma maqueta, nuevos montajes de esta o intervenciones similares son útiles para objetivos terapéuticos de prevención de riesgo de reocurrencia, por ejemplo, situando al niño/a en diversas situaciones 
ficticias que se crean en escena en la maqueta o través de role playing, y que sugieran situaciones de complejidad para promover su capacidad de respuesta con estimulación cognitiva.

Con esto, la técnica también se vuelve útil para trabajar no solo cuestiones relativas a las necesidades culturales que recaen en el tratamiento (interrupción de PAS), sino también para promover el desarrollo del repertorio emocional, cognitivo y conductual, tales como el manejo de la ira, tolerancia, paciencia, sana competencia, trabajo en equipo, creatividad, autoconocimiento, identidad, intereses, roles e identificaciones, estrategias de prevención, etc., o incluso temas más metafísicos o espirituales.

Finalmente, otra analogía interesante de realizar en la maqueta, para promover la conciencia de grupo y la empatía, es mediante la idea de comprender a los individuos como árboles y a las sociedades como bosques que disponen de leyes básicas para su homeostasis. Es así como el niño/a comprende que ningún árbol crece aisladamente, además de poder incluir, con esta analogía, la adquisición de aprendizajes respecto de la diversidad cultural, social y sexual, y tantas otras cosas relativas a la vida de los árboles. Cualquiera sea el contenido analógico, el objetivo es que el niño/a se instruya y se le psicoeduque acerca del entramado social en el cual se encuentra inserto, y adquiera una idea de globalidad, promoviendo una convergencia armoniosa entre una definición clara del sí mismo (identidad) y el desarrollo de empatía y conciencia de grupo. Ello facilitará el logro de un equilibrio ideal entre las necesidades de la cultura y el control y satisfacción de las pulsiones, promoviendo, además, el desarrollo de su conciencia social mediante la adquisición de un ideal cultural de sociedad armoniosa (un mundo perfecto).

Por último, se vuelve crucial trabajar de manera conjunta con los cuidadores en relación con los aprendizajes de los NNA, con el objetivo de que los avances sean reforzados en su contexto natural, por lo que un trabajo familiar y de retroalimentación es crucial para la efectividad del tratamiento. Asimismo, se puede incluir al adulto en las sesiones con la maqueta en todas las formas que puedan ser útiles; por ejemplo, para que sea el niño/a quien se la presente y se la explique a su cuidador/a, una vez que esta ya se ha trabajado de forma individual, o puedan crear un montaje juntos, con ayuda terapéutica. 


\section{Resultados y conclusiones}

En el estudio de este tipo de fenómenos, como el de PAS, en que los límites de los roles de víctima y victimario se vuelven difusos, se deja deja entrever de sobremanera la necesidad de un abordaje social y crítico para comprender su funcionamiento. El estudio del orden del entramado social que envuelve al NNA es siempre primordial y la teoría ecológica se considera muy atingente, sobre todo si se parte de la premisa que las vulneraciones previas explican, en mayor medida, la aparición de conductas de PAS. No obstante, si todas las vulneraciones previas se han detenido y el niño/a ejerce, de todas formas, prácticas abusivas sexuales como respuesta, entonces la conducta trasgresora o infractora se ha asimilado individualmente y es importante analizarla con relación a su motivación intrínseca, es decir, con relación a la significación de ley que opera en él y a la calidad del sentimiento de culpa que le genera la transgresión, dependiendo además de sus capacidades cognitivas y la información que maneja.

Para ello, en el presente escrito se diseñaron intervenciones inspiradas en el análisis de los constructos psicoanalíticos. Este trabajo metodológico se ejecuta desde la premisa que el NNA regulará la satisfacción de sus pulsiones sobre la base de la gratificación de un deseo mayor: el de ser integrado y aceptado, renunciando a sus deseos básicos de gratificación sexual y/o de agresión por el deseo de ser amado e integrado por una cultura que lo comprende y le brinda un tratamiento reparador. En este sentido, el terapeuta se posiciona como un mediador entre las pulsiones del niño y las necesidades de la cultura.

Se considera que las técnicas detalladas entregan inmensa información respecto de aspectos psicológicos del niño y su ambiente, y acerca de dónde y cómo intervenir en determinados niveles de su ecología. En este sentido, se vuelve un aporte para los objetivos de un modelo que interviene en diversas esferas ambientales de las que el niño/a puede dar cuenta en sesión.

De esta manera, la metodología propuesta es perfectamente imbricable dentro de diseños interventivos basados en un enfoque ecológico y multidimensional, ya que, si bien el enfoque psicoanalítico pone los ojos en el individuo, refleja espontáneamente una ideología radicalmente ecológica pero más específica, pues estudia los procesos psíquicos acerca de cómo la cultura opera dentro de la psique y de 
cómo ocurrió ese paso en que, de ser parte de un todo indiferenciado, el niño/o introyecta al mundo dentro de sí, para volverse uno mismo, o, en términos ecológicos, pensar en cómo el niño/a también contiene a los subsistemas dentro de sí mismo y experiencia subjetivamente el estar inmerso en su ambiente, entendiendo que el desarrollo humano se da en la interacción constante entre las variables individuales y ambientales.

Con esto, la presente reflexión teórico-clínica muestra también de qué manera es posible utilizar la creatividad y las habilidades clínicas para realizar intervenciones personalizadas y basadas en la experiencia única que entrega el abordaje del caso.

\section{Discusión}

La pesquisa oportuna de una emisión de PAS y el acceso inmediato a un tratamiento eficaz es un deber social fundamental para detener la cadena de abusos, ya que muchos NNA ejecutores de PAS han sido víctimas de abusos sexuales anteriores, siendo muchos de éstos no visibilizados en su momento por la sociedad, si no es por la repetición patógena actual de prácticas abusivas sexuales desde un rol de victimario, y siendo doblemente vulnerado en relación a la estigmatización que esta práctica conlleva. Si hay algo más vulnerador que el abuso sexual hacia un niño/a, es el abuso sexual que ha sido silenciado y ocultado, emergiendo con ello no solo pulsiones sexuales que el mismo adolescente no controla y no se explica, sino también, en casos más extremos, pulsiones de agresión y venganza hacia una sociedad que lo ha agredido y lo continúa haciendo, en tanto lo castiga y no lo trata.

La cultura, entonces, debe enmendar el daño, brindando un tratamiento reparatorio y no sancionador, aunque ciertamente obligatorio, en el cual al trasgresor sexual, aún juvenil, se le reconoce, primeramente, como una víctima olvidada (incluso para sí mismo), subsanando un ideal de Estado garante de derechos, versus un Estado represor que penaliza aquello que la misma sociedad configura a través de la desigualdad social y la falta de oportunidades que preceden incluso a la concepción.

Por otra parte, para promover la deconstrucción de las desigualdades sociales desde el micromundo de la intervención, se vuelve un de- 
ber ético fomentar que los niños y adolescentes comprendan la importancia de la legalidad como forma de regulación social, brindándoles herramientas para que sean capaces de comprender el poder inherente a la cultura que los rige, en donde el espiritu de la ley sea comprendido en torno a su fin último: el de ser un beneficio cultural equitativo para todos los integrantes de la sociedad, basado en un cuerpo legal que se reactualiza en orden de los cambios y el desarrollo evolutivo de a quienes rige. Por ello, tanto el cumplimiento como el cuestionamiento a la ley son ejercicios fundamentales para una cultura en constante evolución, y que debieran promoverse mientras se ejecuta la terapia infanto-juvenil.

\section{Referencias}

Arsuaga, J. L. y Martínez, I. (1998). La especie elegida. Cap. 11, La inteligencia social; tamaño del cerebro y tamaño del grupo social. Temas de Hoy.

Bronfenbrenner, U. (1987). La ecología del desarrollo humano. Barcelona: Paidós.

Deprode Sename. (2019). Bases técnicas. Programa especializado en intervención con adolescentes que presentan conductas abusivas de carácter sexual. Consultado el día 10 febrero de 2020. Recuperado de https://www.sename.cl/web/wp-content/ uploads/2019/05/Orientaciones-Tecnicas-PAS.pdf

Díaz Morfa, J. (2003). Save the Children, 2000.

Freud, S. (1978). El malestar en la cultura, en Obras completas Volumen XXI. Buenos Aires: Amorrortu Ediciones.

Frías, M., Eréndida, A. y Díaz, S. (2003). Predictores de la conducta antisocial juvenil: un modelo ecológico. Consultado el día 01 de febrero de 2020. Recuperado de https://www.scielo. br/scielo.php?pid=S1413294X2003000100003\&script=sci arttext\#back1

Friedrich, W. N. (2003). Problemas de comportamiento sexual problemático en preadolescentes.

Instituto Nacional de Estadísticas [INE]. (2018). Estadísticas vitales. Informe anual 2016. Consultado el día 01 de febrero de 2020. Recuperado de https:/www.ine.cl/docs/default-source/ demográficas-y-vitales/vitales/anuarios/2016/vitales-2016. pdf?sfvrsn $=15$ 
Klein, M. (1932). El Psicoanalisis de niños, en Obras Completas Vol. II. Editorial Rústica.

ONG Paicaví y Aldeas infantiles. (2014). Guía sobre conductas sexuales problemáticas y prácticas abusivas sexuales. Recuperado de https://www.sos-childrensvillages.org/getmedia/f136fd3c1b47-4522-88ba-dbfa8228b43c/Guide-to-Problematic-Sexual-Behaviours-and-Abusive-Sexual-Practises_Spanish. pdf

Peinado, M. T. (2010). Masturbación en el lactante y en el niño. Revista Anales de Pediatría Continuada de la Asociación Española de Pediatría, 8(5). Consultado el día 20 de julio de 2018. Recuperado dehttp://www.apcontinuada.com/es/masturbacion-el-lactante-el-nino/articulo/80000579/

Pérez-Luco, R., Vásquez, O., Muñoz, M., Álvarez, L. y Bustamante, J. (2015). Simposio “Adolescentes chilenos con prácticas abusivas sexuales: Avances en la conceptualización, evaluación y tratamiento del fenómeno". X Congreso Chileno de Psicología CRUCh. Consultado el día 02 de febrero de 2020. Recuperado de https://www.researchgate.net/publication/322365597_Adolescentes_chilenos_con_practicas_abusivas_sexuales_Avances_en_la_conceptualizacion_ evaluacion_y_tratamiento_del_fenomeno

Pérez-Luco, R., Zambrando, A., Alarcón P. y Alarcón, M. (2014). Manual de evaluación diferenciada. Modelo multudimensional de intervención diferenciada con adolescentes (MMIDA). Constulado el día 02 de febrero de 2020. Recuperado de https://www.academia.edu/14846920/Manual_de_Evaluación_Diferenciada._Modelo_Multidimensional_de_Intervención_Diferenciada_con_Adolescentes_MMIDA_

Valenzuela, V. \& Escudero, L. (2013). Pácticas abusivas sexuales de niñas, niños y adolescentes. Recuperado de http://www. observaderechos.cl/site/wp-content/uploads/2013/12/ Prácticas-Abusivas-Sexuales-de-Niños-Niñas-y-Adolescentes-ONG-PAICABI.pdf 